\title{
Nonequilibrium superconducting thin films with sub-gap and pair-breaking photon illumination
}

\author{
T Guruswamy ${ }^{*} \mathrm{D}$ J Goldie, and S Withington \\ Quantum Sensors Group, Cavendish Laboratory, University of Cambridge, \\ $J$ J Thomson Avenue, Cambridge, CB3 OHE, UK
}

(Dated: February 6, 2015)

\begin{abstract}
We calculate nonequilibrium quasiparticle and phonon distributions for a number of widelyused low transition temperature thin-film superconductors under constant, uniform illumination by sub-gap probe and pair-breaking signal photons simultaneously. From these distributions we calculate material-characteristic parameters that allow rapid evaluation of an effective quasiparticle temperature using a simple analytical expression, for all materials studied ( $\mathrm{Mo}, \mathrm{Al}, \mathrm{Ta}, \mathrm{Nb}$, and $\mathrm{NbN}$ ) for all photon energies. We also explore the temperature and energy-dependence of the low-energy quasiparticle generation efficiency $\eta$ by pair-breaking signal photons finding $\eta \approx 0.6$ in the limit of thick films at low bath temperatures that is material-independent. Taking the energy distribution of excess quasiparticles into account, we find $\eta \rightarrow 1$ as the bath temperature approaches the transition temperature in agreement with the assumption of the two-temperature model of the nonequilibrium response that is appropriate in that regime. The behaviour of $\eta$ with signal frequency scaled by the superconducting energy gap is also shown to be material-independent, and is in qualitative agreement with recent experimental results. An enhancement of $\eta$ in the presence of sub-gap (probe) photons is shown to be most significant at signal frequencies near the superconducting gap frequency and arises due to multiple photon absorption events that increase the average energy of excess quasiparticles above that in the absence of the probe.
\end{abstract}

PACS numbers: 74.40.Gh, 74.78.-w, 29.40.-n, 74.25.N-

\section{INTRODUCTION}

The energy distribution of the quasiparticle excitations of a superconductor determines its electrical ${ }^{1}$ and thermal transport properties. ${ }^{[2]} \mathrm{A}$ theoretical understanding of devices including kinetic inductance detectors (KIDs) $\frac{\sqrt[3]{6} 5}{\sqrt[5]{4}}$ superconducting tunnel junctions, $\frac{\sqrt[6]{6}}{,}$ superconducting qubits, 799 SQUID based parametric amplifiers and mixers, $\frac{10}{10}$ and quantum capacitance detectors ${ }^{11}$ requires a description of the quasiparticle system response. Therefore, a method for calculating the excited, possibly nonequilibrium distribution of quasiparticles is central to any device model.

Nonequilibrium superconducting detectors rely on pairbreaking to create their detected signal. We are particularly interested in thin-film superconductors fabricated on a dielectric substrate that is cooled to a sufficiently low temperature (the bath temperature) $T_{b}$. Typically $T_{b} \sim 0.1 T_{c}$ where $T_{c}$ is the superconducting transition temperature. An interacting signal photon of energy $h \nu_{\text {signal }}$, where $h$ is Planck's constant and $\nu_{\text {signal }}$ the signal frequency, directly breaks a condensate pair in the superconductor provided $h \nu_{\text {signal }} \geq 2 \Delta$ where $\Delta$ is the superconducting energy gap. Due to the high density of states close to the gap, and for moderate energy photons, the interaction creates a distribution of excited quasiparticles with peaks ${ }^{12}$ at $E=\Delta$ and $E=h \nu_{\text {signal }}-\Delta$ where $E$ is the quasiparticle energy. $E$ is related to the underlying Bloch state energy $\epsilon$ by $E=\sqrt{\epsilon^{2}+\Delta^{2}}$. Higher energy signal photons will directly release an atomic electron via the photoelectric effect. In this case effects associated with localised heating and gap-reduction or "hotspots" may become important. In this work we concentrate on modelling the detection of moderate energy photons $h \nu_{\text {signal }}<\Omega_{D}$ where these effects are insignificant. The photon energies considered are particularly important to understand the responsivity, sensitivity and signalto-noise of currently-deployed and planned astronomical instruments performing measurements in the frequency window of 0.1 to $10 \mathrm{THz}$. In the case of KIDs, the nonequilibrium state created by the interaction is monitored using a probe of energy $h \nu_{\text {probe }} \ll 2 \Delta$, where $\nu_{\text {probe }}$ is the probe frequency, so an additional drive term needs to be included in the detailed analysis.

An important consideration in any nonequilibrium superconducting detector operating at low reduced temperatures $T_{b} / T_{c} \ll 1$ is that the detected signal is most influenced by the presence of low-energy quasiparticles because the relaxation of the primary excitation occurs on time-scales that are much shorter than the ultimate relaxation time of the low-energy excess. (Here we define low-energy quasiparticles to have $E<3 \Delta$.) We assume the signal photons interact with $100 \%$ efficiency i.e. we ignore for example any optical coupling efficiencies. A primary excitation with $E=h \nu_{\text {signal }}-\Delta$ relaxes towards the gap emitting a phonon. If this emitted phonon has energy $\Omega \geq 2 \Delta$, an additional pair may be broken enhancing the number of low-energy excess quasiparticles and thus the detected signal. But in a thin-film this emitted phonon may be lost into the substrate, reducing the number of low-energy excitations. The average number $m$ of lowenergy quasiparticles resulting from the interaction of a single high-energy photon can be quantified in terms of a quasiparticle generation efficiency (or quasiparticle yield) $\eta$. If the low-energy quasiparticles have average energy 
$\left\langle E_{q p}\right\rangle$ then the efficiency $\eta=m\left\langle E_{q p}\right\rangle / h \nu_{\text {signal }}$. Often it is assumed $\left\langle E_{q p}\right\rangle=\Delta$. 13

Phonon loss means $\eta \leq 1$. Most existing work assumes a value of $\eta \approx 0.6$ for all materials, photon frequencies and bath temperatures. ${ }^{3}$ At high temperatures $T \approx T_{c}$ our description of the energy-cascade no longer applies $14 \mid 15$ because thermal phonon energies become comparable with $\Delta$, and scattering and recombination occur on comparable timescales. Then a two-temperature model describing the quasiparticle and phonon systems that assumes $\eta=1$ is most appropriate. Here we show that the full nonequilibrium calculation yields $\eta \rightarrow 1$ as $T_{b} / T_{c} \rightarrow 1$ in agreement with the assumption of the two-temperature model.

Previous work modelled the effect of incoming energy at low temperatures with an effective temperature ${ }^{[16}$ or as an effective chemical potential ${ }^{17}$ for the quasiparticle distribution. To go beyond this analysis, Chang and Scalapino 18,19 derived a set of coupled nonlinear kinetic equations describing the interactions of quasiparticles and phonons to find their respective energy distributions $f(E)$ and $n(\Omega)$. They solved for the nonequilibrium distributions resulting from various drive terms, including photon and phonon injection. A number of investigations have explored the effect of very high energy photons (x-ray or optical photons) on infinite superconductors ${ }^{20121}$ and thin films ${ }^{22}$ calculating a quasiparticle generation efficiency of $\eta \approx 0.6$, ignoring $\Omega \geq 2 \Delta$ phonon loss. Another approach ${ }^{13}$ considered the time-evolution following local energy deposition into the thin film. Kozorezov et al. 23 considered the energy downconversion process after absorption of a high energy photon for a variety of materials and concluded that the materials can be categorised into three different classes, with the low energy-gap superconductors all having $\eta \approx 0.6$.

We have previously reported ${ }^{24}$ a numerical approach that solves the coupled kinetic equations describing the quasiparticle and phonon distributions $f(E)$ and $n(\Omega)$ for a superconducting thin-film driven by a sub-gap probe, 24 and including the effect of an additional pair-breaking signal, ${ }^{25}$ for Al thin-films at low temperatures $T \sim 0.1 T_{c}$. We have also reported detailed calculations of KID characteristics (quality factors and quasiparticle lifetimes as functions of readout power and $T_{b}$ ) that were compared to precise measurements of $\mathrm{Al}$ resonator behaviour finding good agreement.26 More recent measurements on $\mathrm{KIDs}^{27}$ of the quasiparticle number and recombination time-dependence on readout power appear to show that they must be explained using nonequilibrium quasiparticle distributions. Other recent measurements with a Ta KID ${ }^{28}$ report a detector response that has the same qualitative features as the energy dependence of $\eta$ for $\mathrm{Al}$ described in Guruswamy et al. ${ }^{\sqrt[25]{3}}$ Here we apply the method to a number of other technologically important low temperature superconductors and discuss the physics involved. We concentrate in particular on the refractory elements and extend the earlier analysis to bath temperatures approaching the superconducting critical temperature $T_{c}$. The highest probe powers studied here
( $\sim 10^{8} \mathrm{~W} \mathrm{~m}^{-3}$ in $\left.\mathrm{Nb}\right)$ approach those that we estimate were used by Chin et al. ${ }^{29}$ In that work high absorbed (probe) power densities were associated with large induced supercurrents and magnetic fields approaching the critical field. Over most of the range of powers that we study our calculations show that these effects are negligible. Nevertheless we include high absorbed powers in our modelling to enter this regime. We study Mo, Al, Ta (in its higher $T_{c} \sim 4.4 \mathrm{~K}$ form), $\mathrm{Nb}$ and $\mathrm{NbN}$. In each instance we use measured material properties that characterise films that we deposit by sputtering under ultra-high-vacuum. We study thin films with thicknesses in the range 40 to $500 \mathrm{~nm}$ that are most commonly used for KIDs. Application of our results to ultrathin films $(d \sim 10 \mathrm{~nm})$ must be done with caution, but even so we expect them to be representative. Our results are applicable to many different device designs, as only the phonon escape time to the substrate $\tau_{l}$ is device-geometry dependent. The method assumes that phonon pair-breaking, quasiparticle recombination, and electron-phonon scattering are the only significant interaction processes. This assumption is investigated in section IIB for typical thin-films intended for detector applications. Section II reviews superconducting parameters for the materials discussed, considers the relative contribution from electron-electron scattering for the different materials, describes the numerical method, and also describes how $\eta$ is calculated for a pair-breaking signal. Section III presents an analytical superconductor cooling model that can be used to calculate the effective quasiparticle temperature $T_{N}^{*}$ for both sub-gap and pair-breaking photons. Section [V] describes detailed numerical results for both sub-gap and pair-breaking photon interactions including the effect of the effect of changes in the bath temperature. Section $\mathrm{V}$ summarises the work done.

\section{METHODS}

\section{A. Superconducting parameters}

The superconducting parameters needed for modelling are the characteristic quasiparticle lifetime

$$
\tau_{0}=\frac{Z_{1}(0) \hbar}{2 \pi b\left(k_{B} T_{c}\right)^{3}},
$$

and the characteristic phonon lifetime

$$
\tau_{0}^{\phi}=\frac{\hbar N_{\text {ion }}}{4 \pi^{2} N(0)_{\mathrm{bs}}\left\langle\alpha^{2}\right\rangle \Delta_{0}},
$$

as defined by Kaplan et al. ${ }^{30} N(0)$ and $N(0)_{\text {bs }}$ are the mass-enhanced and bare single-spin density of states at the Fermi energy $\epsilon_{F}$ respectively, where $N(0)=$ $Z_{1}(0) N(0)_{\text {bs }}, N_{\text {ion }}$ is the ion density, and $\Delta_{0}$ the zero temperature superconducting energy gap. $Z_{1}(0)$ is the electron-phonon renormalisation factor $Z_{1}(0)=1+\lambda$, where $\lambda=2 \int_{0}^{\infty} \mathrm{d} \Omega \alpha^{2}(\Omega) F(\Omega) / \Omega$ is the dimensionless electron-phonon coupling strength. $\alpha^{2}(\Omega) F(\Omega)$ is the 
Eliashberg function, $\alpha^{2}(\Omega)$ is the electron-phonon interaction matrix element and $F(\Omega)$ is the phonon density of states. The material-dependent parameter $b$ is calculated within a Debye model such that $b \Omega^{2}=\alpha^{2}(\Omega) F(\Omega)$ for low phonon energies $\Omega \sim 2 \Delta_{0}$. The averaged value of the interaction matrix element is $\left\langle\alpha^{2}\right\rangle=1 / 3 \int_{0}^{\infty} \mathrm{d} \Omega \alpha^{2}(\Omega) F(\Omega)$. Values of $b$ and $\left\langle\alpha^{2}\right\rangle$ for Al, Ta and $\mathrm{Nb}$ are already tabulated. $\stackrel{30}{-}$ For Mo we use the point-contact measurements of Caro et al. $\stackrel{31}{ }$ while for $\mathrm{NbN}$ we use the tunnelling data of Kihlstrom et al. ${ }^{32}$ and the transport property measurements of Semenov et al. 33 We note that the precision of our calculations of $b$ are dependent on the accuracy within which we can interpret the relevant low-frequency parts of the reported $\alpha^{2}(\Omega) F(\Omega)$ data. We assume here that the phonons of the superconducting film can be described by a 3 -dimensional (3-D) model. For ultrathin films, typically of thickness $d<10 \mathrm{~nm}$, this assumption fails and measured values of the temperature dependence of the normal-state electron-phonon relaxation time $\tau_{e-p}$ indicate a reduced phonon dimensionality 34

\begin{tabular}{lrrrrr}
\hline Material & $\mathrm{Mo}$ & $\mathrm{Al}$ & $\mathrm{Ta}$ & $\mathrm{Nb}$ & $\mathrm{NbN}$ \\
\hline$\Delta_{0}(\mu \mathrm{eV})$ & 140 & 180 & 700 & 1470 & 2560 \\
$\nu_{\text {gap }}(\mathrm{GHz})$ & 68 & 87 & 339 & 711 & 1240 \\
$T_{c} / \mathrm{K}$ & 0.92 & 1.18 & 4.6 & 9.67 & 16.8 \\
$b\left(10^{-4} \mathrm{meV}^{-2}\right)$ & 2.28 & 3.17 & 17.3 & 40 & 47 \\
$\left\langle\alpha^{2}\right\rangle(\mathrm{meV})$ & 1.62 & 1.93 & 1.38 & 4.6 & 4.99 \\
$\lambda$ & 0.42 & 0.43 & 0.69 & 1.84 & 1.46 \\
$\tau_{0}(\mathrm{~ns})$ & 1310 & 438 & 1.78 & 0.15 & 0.02 \\
$\tau_{0}^{\phi}(\mathrm{ps})$ & 231 & 260 & 22.7 & 4.17 & 5.98 \\
$\tau_{\text {ec }}^{\phi} / \tau_{0}^{\phi}$ & 1.04 & 1.04 & 0.91 & 0.94 & 1.01 \\
\hline
\end{tabular}

Table I. Characteristic quasiparticle and phonon lifetimes, and associated parameters. Data from Gladstone et al. ${ }^{[5]}$, Kaplan et al. ${ }^{[30}$ or Zehnder ${ }^{13}$ unless otherwise specified. For Mo and $\mathrm{NbN}$ we use measurements of $\alpha^{2}(\Omega) F(\Omega)$ from Caro et al. $\frac{31}{31}$ and Kihlstrom et al. ${ }^{32}$ respectively. $\tau_{\mathrm{ec}}^{\phi}$ is the value of $\tau_{0}^{\phi}$ required for energy conservation calculated from (8). $\nu_{\text {gap }}=2 \Delta_{0} / h$ is the gap frequency.

The characteristic times calculated are listed in table I. We assume the weak-coupling relationship $\Delta_{0}=$ $1.76 k_{B} T_{c}$ for all materials to determine $T_{c}$ in table $\mathbb{I}$. The assumption is reasonable for $\mathrm{Mo} \mathrm{Al}$ and $\mathrm{Ta}$, but gives a slightly higher $T_{c}$ compared to experiment for Nb (typically $\left.T_{c} \sim 9.3 \mathrm{~K}\right)$ and for $\mathrm{NbN}\left(T_{c} \sim 15 \mathrm{~K}\right)$. However we do not expect the assumption to significantly affect the physics of the conclusions drawn; the differences introduced by strong coupling are reduced because of the short lifetimes of states with energy $E \gg \Delta$ ! $180^{\phi} \tau_{\mathrm{ec}}^{\phi}$ is the value of $\tau_{0}^{\phi}$ required for energy conservation calculated from (8) and discussed in more detail in section II C, The values of $\tau_{0}$ for $\mathrm{Al}$, Ta and $\mathrm{Nb}$ shown in table $\mathrm{I}$ have already been widely used to interpret measurements, often of quasiparticle recombination times. For $\mathrm{NbN}$ there is less available experimental data. Gousev et al. $\underline{36}$ measured $\tau_{e-p}$ in ultrathin $(3.5 \mathrm{~nm}) \mathrm{NbN}$ films finding $\tau_{e-p} \simeq 35 \mathrm{ps}$ for $T=5 \mathrm{~K}$ and with temperature depen- dence $\tau_{e-p} \sim T^{1.6}$. Scaling this measurement to $16.8 \mathrm{~K}$ we estimate $\tau_{e-p} \sim 5 \mathrm{ps}$. Identifying ${ }^{\sqrt{30}} \tau_{e-p}=\tau_{0} / 8.4$, a 3 -D phonon spectrum gives a value of $2.4 \mathrm{ps}$ in reasonable agreement.

\section{B. Electron-electron vs electron-phonon scattering}

Insight into the contribution from electron-electron $e-e$ scattering in the down-conversion process is most easily found by considering the relevant scattering rates $T_{c}$. For the electron energies of interest $\epsilon \ll \Omega_{D}$, we assume that electron-phonon $(e-p)$ inelastic scattering dominates over $(e-e)$ scattering. We previously investigated ${ }^{25}$ to what extent this assumption is valid for low-resistivity $\mathrm{Al}$ films. Now we consider the other low- $T_{c}$ superconductors using material parameters typical of those that we measure experimentally for the thin-films we deposit by magnetronsputtering in ultra-high vacuum.

In the normal-state the $e-p$ scattering rate $\tau_{e-p}^{-1}$ is 37

$$
\tau_{e-p}^{-1}(\epsilon)=\frac{2 \pi}{\hbar} \int_{0}^{\epsilon} \mathrm{d} \Omega \alpha^{2}(\Omega) F(\Omega)
$$

Assuming $\alpha^{2}(\Omega)$ is constant at low energies, a Debye model for phonons $\sqrt{30}$ leads to

$$
\tau_{e-p}^{-1}(\epsilon)=\left\{\begin{array}{ll}
\frac{\epsilon^{3}}{3 \tau_{0}\left(k_{B} T_{c}\right)^{3}} & \text { if } \epsilon<\Omega_{D} \\
\frac{\Omega_{D}^{3}}{3 \tau_{0}\left(k_{B} T_{c}\right)^{3}} & \text { if } \epsilon \geq \Omega_{D}
\end{array} .\right.
$$

The electron-electron scattering rate in clean films is estimated with the Landau-Pomeranchuk formula ${ }^{23}$

$$
\tau_{e-e}^{-1}(\epsilon)=\frac{\epsilon^{2}}{\hbar \epsilon_{F}} \frac{r_{s}^{1 / 2}}{7.96},
$$

where $r_{s}$ is the radius containing one electron charge divided by the Bohr radius (approximated as 1). For thin resistive films the $e-e$ rate can be significantly enhanced so that ${ }^{38}$

$$
\tau_{e-e}^{-1}\left(\epsilon, R_{s q}\right)=\frac{e^{2} R_{s q} \epsilon}{2 \pi^{2} \hbar^{2}} \ln ^{-1} \frac{\hbar \pi}{e^{2} R_{s q}},
$$

where $R_{s q}=\rho / d$ is the sheet resistance of the thin-film, $\rho$ is the resistivity and $d$ is the thickness 39 These normalstate calculations significantly overestimate the $e-e$ scattering rates in the superconducting state at low temperatures $T_{b} \ll T_{c}{ }^{[40]}$ Figure 1 plots the normal-state scattering rates for a $40 \mathrm{~nm}$ thick Mo film and we have used the thin-film resistive $e-e$ scattering rate (6). We identify the following characteristic energies for each material: $\epsilon_{F}$; $\Omega_{D}$; and a low-energy crossover $E_{c}$, below which the $e-e$ scattering rate exceeds the $e-p$ rate. $E_{c} / \Delta$ is highest for Mo for the material parameters chosen, as shown in table [I]. 


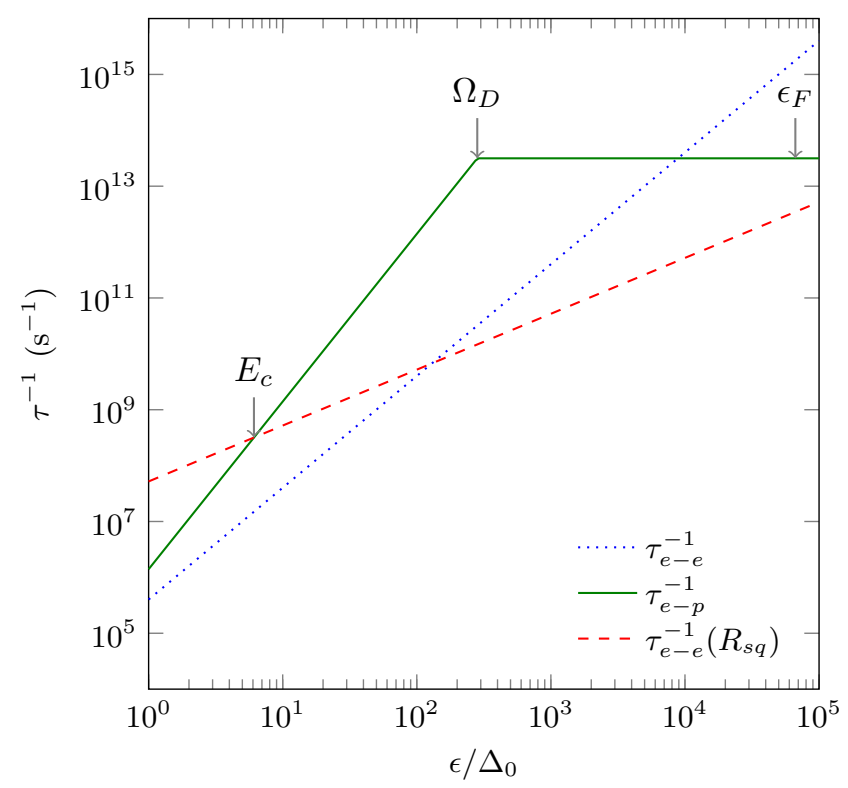

Figure 1. Energy dependence of normal state scattering rates in Mo. Electron-phonon rate $\tau_{e-p}^{-1}(\epsilon)$ (solid green), clean limit electron-electron rate $\tau_{e-e}^{-1}(\epsilon)$ (dotted blue), and thinfilm resistive $\tau_{e-e}^{-1}\left(\epsilon, R_{s q}\right)$ (dashed red). Calculations are for a $40 \mathrm{~nm}$ Mo film with $\rho=9.2 \times 10^{-8} \Omega \mathrm{m}$.

\begin{tabular}{lcccll}
\hline Material & $\rho\left(10^{-8} \Omega \mathrm{m}\right)$ & $d(\mathrm{~nm})$ & $\epsilon_{F}(\mathrm{eV})$ & $\Omega_{D}(\mathrm{meV})$ & $E_{c} / \Delta_{0}$ \\
\hline Mo & 9.2 & 40 & 9.32 & 39.6 & 6.1 \\
$\mathrm{Al}$ & 0.8 & 35 & 11.6 & 36.2 & 1.42 \\
$\mathrm{Ta}(\mathrm{bcc}$, epi) & 4.1 & 100 & 9.5 & 20.7 & 0.23 \\
$\mathrm{Nb}$ & 8.8 & 100 & 6.18 & 23.7 & 0.14 \\
$\mathrm{NbN}{ }^{42143}$ & 250 & 100 & 15.6 & 28.4 & 0.3 \\
\hline
\end{tabular}

Table II. Characteristic material energies related to scattering. $E_{c}$ is the energy at which the electron-phonon scattering rate becomes greater than the thin-film resistive electronelectron scattering rate. Data from Gladstone et al. ${ }^{35}$, Kaplan et al. ${ }^{[0]}$ or Kozorezov et al. ${ }^{[23}$ unless otherwise referenced. The material resistivities and thicknesses used are measured values from thin-films deposited by our group. All data is for polycrystalline films except for Ta where we assume parameters typical of the higher- $T_{c}$ bcc form.

During the energy down-conversion, the inelastic scattering rate is only relevant above energies $E \geq 3 \Delta$ where additional pair breaking is possible. We find that $E_{c}$ is below $3 \Delta$ for all materials except Mo. Figure 1 shows that $e-p$ scattering dominates in that case for $\epsilon \approx 6 \Delta$ to $10^{4} \Delta$ for the modelled Mo film, and $e-e$ scattering may contribute above and below this energy range. However, the $e-e$ scattering rate is considerably suppressed in the superconducting state at low $T / T_{c} \cdot{ }^{40]}$ We conclude that there is negligible contribution from $e-e$ scattering for most of these materials, thicknesses, temperatures and signal photon energies. For Mo we note that additional $e-e$ scattering may increase the quasiparticle generation efficiency over the results presented here by providing an additional pair-breaking mechanism especially near $T_{c}$.

\section{Numerical method}

We solved the coupled kinetic equations ${ }^{18}$ describing the quasiparticle and phonon distributions numerically to find the steady-state driven distributions, $f(E) n(\Omega)$ for the quasiparticles and phonons respectively, using NewtonRaphson iteration. Drive terms for pair-breaking and subgap photons ${ }^{44}$ were included as necessary and the numeric pre-factors of these terms scaled to match the absorbed powers, $P_{\text {probe }}$ for sub-gap photons and $P_{\text {signal }}$ for the pair-breaking signal. The numerical method was iterated until the solutions conserved energy to within $\xi=10^{-5}$. This was quantified as $\xi=\sqrt{\xi_{q p-\phi}^{2}+\xi_{\phi-b}^{2}}$, where $\xi_{q p-\phi}$ is the relative difference between the photon power absorbed and power flow from quasiparticles to phonons, and $\xi_{\phi-b}$ is the relative difference between the photon power absorbed and the power flow from phonons to substrate (held at a temperature $T_{b}$ ). The numerical method is outlined in more detail in Goldie and Withington. ${ }^{24}$

For each material, the temperature dependent energy gap $\Delta(T)$ was calculated using the BCS gap equation,

$$
\frac{1}{N(0) V_{\mathrm{BCS}}}=\int_{\Delta(T)}^{\Omega_{D}} \mathrm{~d} E \frac{1-2 f(E, T)}{\sqrt{E^{2}-\Delta(T)^{2}}},
$$

where $f(E, T)=\left(\exp \left(E / k_{B} T\right)+1\right)^{-1}$ is the Fermi-Dirac distribution, and $N(0) V_{\mathrm{BCS}}$ is the dimensionless electronphonon coupling constant, calculated from the zero temperature form of (7) as $N(0) V_{\mathrm{BCS}}=1 / \sinh ^{-1}\left(\Omega_{D} / \Delta_{0}\right)$.

The numerical method converges on a solution that minimises $\xi$ provided the material parameters satisfy

$$
\frac{2 \pi N(0) \tau_{0}^{\phi} \Delta_{0} \Omega_{D}^{3}}{9 N_{\text {ion }} \tau_{0}\left(k_{B} T_{c}\right)^{3}}=1,
$$

a consequence of matching the low energy part of the measured Eliashberg function to a Debye-model approximation that determines $b$. In our previous work we ensured that $T_{c}$ satisfied $\left(8, \frac{24}{24}\right.$ but then the $T_{c}$ obtained did not precisely agree with (7) (although the difference is small $<1 \%$ ). In this work, since we are also interested in the temperature dependence of the response we calculated $T_{c}$ from (7) and defined an energy-conserving characteristic phonon lifetime $\tau_{\mathrm{ec}}^{\phi}$ to satisfy (8). Given the difficulty of interpreting the low-energy $\alpha^{2}(\Omega) F(\Omega)$ data that we and indeed Kaplan et al. ${ }^{30}$ and others have noted we consider this approach entirely reasonable. The ratios $\tau_{\text {ec }}^{\phi} / \tau_{0}^{\phi}$ are given in table I. Satisfyingly the ratios are within $10 \%$ of unity for all materials studied. In our calculation of $b$ for Mo, our error would be of this order given the difficulty of determining the low-energy $\alpha^{2}(\Omega) F(\Omega)$ from the available data. We note that other estimates of the characteristic times exist (see for example Parlato et al. ${ }^{45}$ ) although those estimates do not necessarily satisfy the energy-conserving requirement. 
In the numerical code the energy-bin distribution for both the quasiparticles and phonons was also changed when calculating bath temperature dependencies. As $T_{b}$ is increased, the phonon-bath power flow $P(\Omega)_{\phi-b}$ above $\Omega=2 \Delta$ increasingly resembles the difference between two thermal distributions, and an increasing fraction of power is carried by high energy phonons, as shown in figure 2. Therefore at high temperatures, the phonon distribution must be accurately calculated to these higher energies. The energy of the last phonon bin $\Omega_{\max }$ significantly affects the energy conservation of the solutions. Figure 2 (inset) shows that to have a relative power flow difference $\xi_{\phi-b}<10^{-5}$, required $\Omega_{\max }>15 k_{B} T_{b}$ for $T_{b} / T_{c}=0.95$.

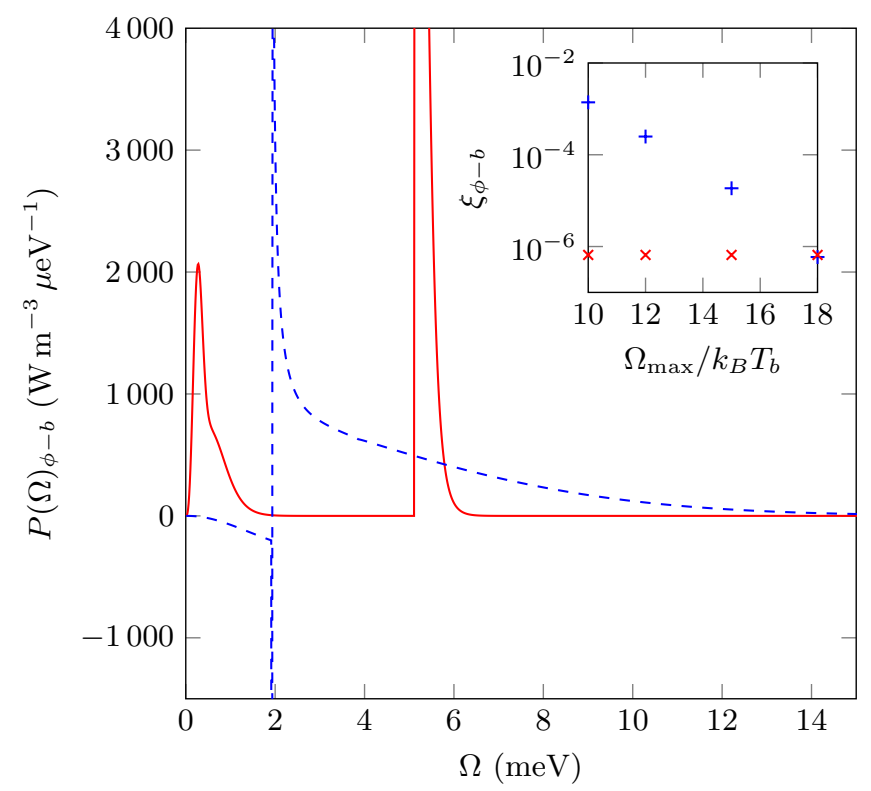

Figure 2. Phonon-bath power flow $P(\Omega)_{\phi-b}$, at $T_{b}=0.1 T_{c}$ (solid red) and $T_{b}=0.95 T_{c}$ (dashed blue). Both for NbN, $P_{\text {probe }}=2 \times 10^{6} \mathrm{~W} \mathrm{~m}^{-3}, P_{\text {signal }}=0, \tau_{l} / \tau_{0}^{\phi}=1$, and $h \nu_{\text {probe }}=$ $16 \mu \mathrm{eV}$. Inset of relative phonon-bath power flow difference $\xi_{\phi-b}$ against the energy of the maximum bin $\Omega_{\max } / k_{B} T_{b}$, at the same bath temperatures $T_{b}=0.1 T_{c}(\operatorname{red} \times)$ and $T_{b}=$ $0.95 T_{c}$ (blue + ).

However, the bin width could not be made too large as the energy gap $\Delta(T)$, readout photon energy $h \nu_{\mathrm{p}}$, and signal photon energy $h \nu_{\mathrm{s}}$ had to be rounded to the nearest multiple of the bin width, such that the photon-induced peaks in the distributions occurred within well-defined energy bins. We chose to model the phonons with $N=2500$ uniformly-sized bins with maximum phonon energy $\Omega_{\max }$, and similarly the quasiparticles with maximum energy $E_{\max }=\Omega_{\max }+\Delta(T)$. Bin width was therefore variable, depending on material and temperature. The quasiparticle density of states broadening parameter $\gamma$ was recalculated for each simulation, changing for different material and bin width combinations. It was chosen to minimise the difference between the quasiparticle number calculated as the integral $4 N(0) \int_{\Delta}^{\infty} \mathrm{d} E \rho_{\mathrm{BCS}}(E, \Delta) f\left(E, T_{b}\right)$, where $\rho_{\mathrm{BCS}}(E, \Delta)=E / \sqrt{E^{2}-\Delta^{2}}$, and the equivalent sum over the discretised distribution with the broadened density of states calculated with $E \rightarrow E+i \gamma$. In all cases the approach worked well.

\section{Low-energy quasiparticle generation efficiency for a pair-breaking signal}

For a single pair-breaking photon we have defined a lowenergy generation efficiency $\eta=m\left\langle E_{q p}\right\rangle / h \nu_{\text {signal }}$. Here we describe the calculation of $\eta$ for a constant input signal power $P_{\text {signal }}$ in a thin-film that may also be driven by a non-direct pair-breaking constant readout power $P_{\text {probe }}$ (such as used for KID readout). The absorbed power creates a number of excess quasiparticles $N_{\text {excess }}=$ $N_{\text {signal }}-N_{\text {probe }}$ such that the quasiparticle system total energy has increased by $E_{\text {excess }}=E_{\text {signal }}-E_{\text {probe }}$, where $N_{\text {signal }}$ and $E_{\text {signal }}$ are the quantities for distributions driven by the signal and probe together. In the steady state, photons absorbed at a rate $\Gamma_{\Phi}$ create low-energy quasiparticles at a rate $\Gamma_{q p}=m \Gamma_{\Phi}$.

Solving a modified set of Rothwarf-Taylor rate equations ${ }^{46}$ for $\Gamma_{\Phi}$ and $\Gamma_{q p}$ results in 25

$$
\eta=\left\langle E_{q p}\right\rangle \frac{\left(N_{\text {signal }}^{2}-N_{\text {probe }}^{2}\right)}{P_{\text {signal }}} \frac{2 R}{1+\beta \tau_{l}},
$$

where $R$ and $\beta$ are the distribution-averaged recombination rate and pair-breaking rates respectively. We define $\left\langle E_{q p}\right\rangle$ the average energy of the excess low-energy quasiparticles where

$$
\left\langle E_{q p}\right\rangle=\frac{\int_{0}^{\infty} E \rho(E)(f(E)-f(E, T)) \mathrm{d} E}{\int_{0}^{\infty} \rho(E)(f(E)-f(E, T)) \mathrm{d} E} .
$$

At low temperatures $\left\langle E_{q p}\right\rangle \approx \Delta$ as usually assumed: taking account of the detailed energy distributions allows $\eta$ to be determined at arbitrary temperatures.

\section{SUPERCONDUCTOR COOLING MODEL}

We used the steady-state driven distributions that were calculated to determine parameters for the thin-film superconductor cooling model outlined in Goldie and Withington. ${ }^{24}$ This model is an analytic expression relating $T_{N}^{*}$ to the power flow $P$ between the quasiparticle and phonon systems (the latter assumed to be the substrate phonons) for a given $T_{b} \ll T_{c}$ and material.

$$
\begin{aligned}
& P=\frac{1}{\eta(P, \nu)} \frac{\Sigma_{s}}{1+\tau_{l} / \tau_{p b}} \times \\
& {\left[T_{N}^{*} \exp \left(\frac{-2 \Delta\left(T_{N}^{*}\right)}{k_{B} T_{N}^{*}}\right)-T_{b} \exp \left(\frac{-2 \Delta\left(T_{b}\right)}{k_{B} T_{b}}\right)\right] .}
\end{aligned}
$$


$\Sigma_{s}$ is a material-dependent constant, $\tau_{p b}$ is the phonon pair breaking time, $\sim \tau_{0}^{\phi}$ for $T_{b} \ll T_{c}$. $T_{N}^{*}$ is the effective temperature calculated to characterise the driven nonequilibrium quasiparticle distributions so that $N_{\mathrm{qp}}=$ $4 N(0) \int_{\Delta}^{\infty} \mathrm{d} E \rho(E) f\left(E, T_{N}^{*}\right) \cdot \eta(P, \nu)$ depends on the drive (probe or pair-breaking signal). Goldie and Withington ${ }^{24}$ showed for a sub-gap probe $\eta\left(P, \nu_{\text {probe }}\right) \equiv \eta_{2 \Delta}$ the fraction of phonon-bath power flow carried by excess phonons with energy $\Omega>2 \Delta\left(T_{b}\right)$ that depends on $P, \nu_{\text {probe }}$ and $\tau_{l}$. Here we show that (11) can also be applied for direct pair-breaking when $\eta\left(P, \nu_{\text {signal }}\right) \equiv \eta$ as defined in section IID

\section{RESULTS}

\section{A. Effects of a sub-gap probe}
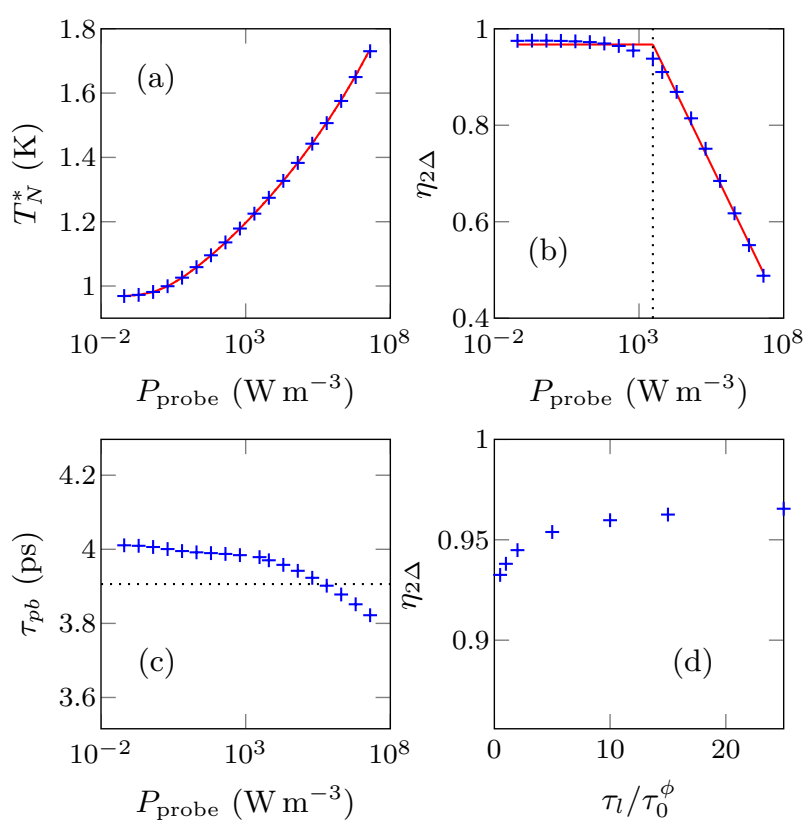

Figure 3. (a) Effective quasiparticle temperatures $T_{N}^{*}$ as a function of $P_{\text {probe }}$; full nonequilibrium calculation (blue + ), and a fit using (11) (red solid line). (b) Fraction of phononbath power flow carried by $\Omega>2 \Delta$ excess phonons as a function of $P_{\text {probe }}$; full nonequilibrium calculation (blue + ), and piecewise fit (red line) to 12 . The dotted vertical line marks $P_{0}$. (c) Distribution-averaged phonon pair-breaking time $\tau_{p b}$. The characteristic phonon lifetime $\tau_{0}^{\phi}$ is marked with the dotted line. (d) $\eta_{2 \Delta}$ as a function of phonon escape time ratio $\tau_{l} / \tau_{0}^{\phi}$. The calculations are for $\mathrm{Nb}$, with $P_{\text {probe }}=$ $2 \times 10^{3} \mathrm{~W} \mathrm{~m}^{-3}, h \nu_{\text {probe }}=16 \mu \mathrm{eV}$, and $\tau_{l} / \tau_{0}^{\phi}=1$.

Here we show and discuss results for modelling of the effect of a sub-gap probe. The data-points (crosses) in figure $3(\mathrm{a})$ show the calculated $T_{N}^{*}$ from the full non-equilibrium solution as a function of $P_{\text {probe. The }}$ solid line shows $T_{N}^{*}$ evaluated with (11) and from this we also calculate $\Sigma_{s}$. The calculations are for $\mathrm{Nb}$, with $P_{\text {probe }}=2 \times 10^{3} \mathrm{~W} \mathrm{~m}^{-3}, h \nu_{\text {probe }}=16 \mu \mathrm{eV}$, and $\tau_{l} / \tau_{0}^{\phi}=1$. The analytical model is an excellent approximation to the full nonequilibrium calculation provided the power-dependence of $\eta_{2 \Delta}$ shown in figure 3(b) is taken into account. The solid line in figure $3(\mathrm{~b})$ is a piecewise fit such that

$$
\eta_{2 \Delta}(P)= \begin{cases}\eta_{0} & \text { if } P \leq P_{0} \\ \eta_{1} \ln \left(P / P_{0}\right)+\eta_{0} & \text { if } P>P_{0}\end{cases}
$$

$P_{0}$ characterises a "knee" probe power below which $\eta_{2 \Delta}$ is constant and $\eta_{1}$ characterises the energy dependence of $\eta_{2 \Delta}$ at higher probe powers. Both are material dependent. Figure $3(\mathrm{c})$ shows that $\tau_{p b} \sim \tau_{0}^{\phi}$ is a good approximation over the range of interest. Figure $3(\mathrm{~d})$ shows that $\eta_{2 \Delta}$ has no significant dependence on the phonon escape time $\tau_{l}$.
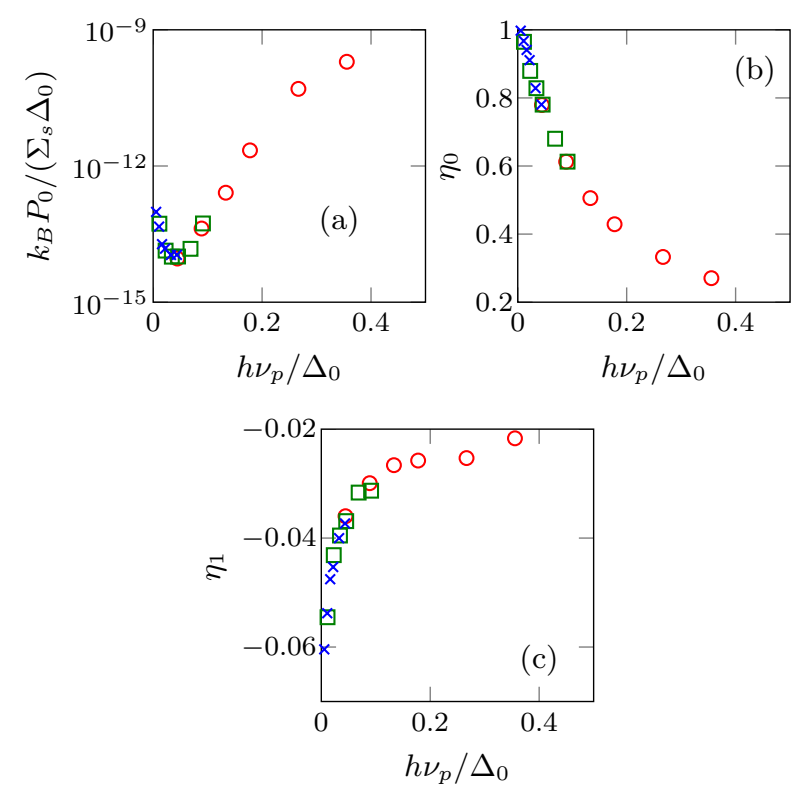

Figure 4. Parameters for piecewise fits to $\eta_{2 \Delta}$ for $\mathrm{Nb}$ (blue $\times$ ), Ta (green $\square$ ), and $\mathrm{Al}$ (red $\bigcirc$ ) calculated at a range of frequencies $h \nu_{\text {probe }}$ (a) $P_{0}$ against scaled frequency. (b) $\eta_{0}$ (constant section) against scaled frequency. (c) $\eta_{1}$ (slope for linear section) against scaled frequency.

The dependence of the fit parameters $P_{0}, \eta_{0}$, and $\eta_{1}$ on probe photon energy is shown in figure 4. This includes calculations for $\mathrm{Nb}($ blue $\times$ ), Ta (green $\square$ ), and $\mathrm{Al}$ (red $\bigcirc$ ). Scaling the energy of the photon by $\Delta_{0}$ demonstrates the monotonic behaviour for all the materials. $P_{0}$ shown in figure $4(\mathrm{a})$ is scaled by the material-dependent $\Sigma_{s}$ to emphasise the commonality.

Table III summarises parameters derived from the modelling to describe sub-gap photon interactions in all of the materials studied. The table shows $\Sigma_{s}$ and knee parameter $P_{0}$ at $h \nu_{\text {probe }}=16 \mu \mathrm{eV}$ and $\tau_{l} / \tau_{0}^{\phi}=1$. The values of $\Sigma_{s}$ shown can also be used for estimates of $T_{N}^{*}$ for a 


\begin{tabular}{lcll}
\hline Material & $\Delta_{0}(\mu \mathrm{eV})$ & $\Sigma_{s}\left(\mathrm{~W} \mathrm{~m}^{-3} \mathrm{~K}^{-1}\right)$ & $P_{0}\left(\mathrm{~W} \mathrm{~m}^{-3}\right)$ \\
\hline $\mathrm{Mo}$ & 140 & $1.42 \cdot 10^{10}$ & $2.96 \cdot 10^{-3}$ \\
$\mathrm{Al}$ & 180 & $3.23 \cdot 10^{10}$ & $2.83 \cdot 10^{-3}$ \\
$\mathrm{Ta}$ & 700 & $1.17 \cdot 10^{14}$ & $1.30 \cdot 10^{1}$ \\
$\mathrm{Nb}$ & 1470 & $3.83 \cdot 10^{15}$ & $3.02 \cdot 10^{3}$ \\
$\mathrm{NbN}$ & 2560 & $1.29 \cdot 10^{16}$ & $3.17 \cdot 10^{4}$ \\
\hline
\end{tabular}

Table III. Superconductor cooling model material parameter $\Sigma_{s}$, and knee power $P_{0}$ at $h \nu_{\text {probe }}=16 \mu \mathrm{eV}$ and $\tau_{l} / \tau_{0}^{\phi}=$ 1 , obtained from fits to results of the full nonequilibrium calculation.

direct pair-breaking signal as we will discuss in the next section with an appropriate energy-dependent $\eta$.

\section{B. Effects of direct pair-breaking}

Here we discuss results for a direct pair-breaking signal with $2 \Delta<h \nu_{\text {signal }}<10 \Delta$. Figure 5 shows how $\eta$

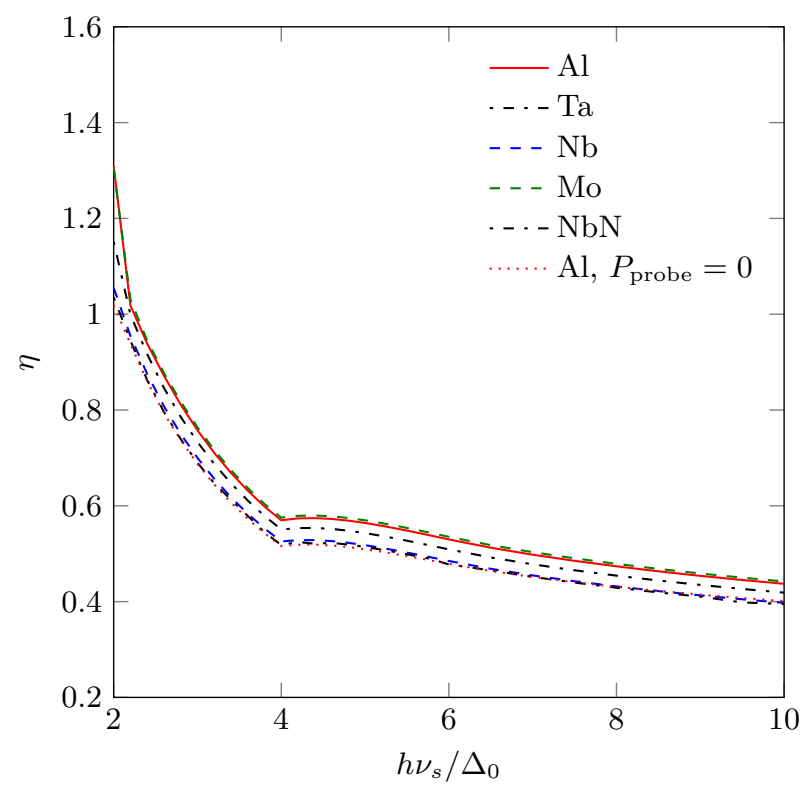

Figure 5. Quasiparticle generation efficiency $\eta_{\text {power against sig- }}$ nal photon energy $h \nu_{\text {signal }}$ for different materials. Calculated for $T_{b}=0.1 T_{c}, P_{\text {probe }}=2 \times 10^{3} \mathrm{~W} \mathrm{~m}^{-3}, h \nu_{\text {probe }}=16 \mu \mathrm{eV}$, $P_{\text {signal }}=2 \mathrm{~W} \mathrm{~m}^{-3}$, and $\tau_{l} / \tau_{0}^{\phi}=1$.

varies with signal photon energy. $\eta$ decreases from unity in the range $h \nu_{\text {signal }}=2 \Delta$ to $4 \Delta$, as phonons emitted by quasiparticles scattering towards the gap escape the thin film. Above $h \nu_{\text {signal }}=4 \Delta$, the phonons emitted in scattering can break additional Cooper pairs, increasing the quasiparticle generation efficiency. The magnitude of the increase depends on the phonon trapping factor. 25 Measurements of the response of a Ta KID ${ }^{28}$ at signal frequencies close to the gap energy show these characteristic features. The figure shows that when $h \nu_{\text {signal }}, T_{b}$ and $\tau_{l}$ are scaled by the relevant material parameters, the quasiparticle generation efficiency is the same for all materials. Kozorezov et al. ${ }^{23}$ predicted that the quasiparticle generation efficiency could be categorised into three classes of superconductors. The superconductors included in this work, which fall within either the second $(\mathrm{Ta}, \mathrm{Nb}, \mathrm{NbN})$ or third (Al, Mo) class, have a materialindependent $\eta \approx 0.6$. This conclusion differs somewhat from that of Zehnder. ${ }^{13}$ We understand this as due to the current work examining the steady-state response to constant incoming power, and scaling all relevant parameters by material characteristics, whereas Zehnder's work examined the time-dependent response including quasiparticle out-diffusion from a localised hot-spot, and chose a fixed cutoff time of $10 \mathrm{~ns}$ to calculate the quasiparticle generation efficiency unscaled by material. We emphasise that for the low energy photons considered in the present work localised hot-spot formation (and consequent gap suppression) does not occur for the signal powers studied.

The other effect shown in figure 5 is the enhancement of $\eta$ by the readout power due to an increase in the average energy of generated quasiparticles. For $h \nu_{\text {signal }} \simeq 2 \Delta$, it can be seen that $\eta>1$ which may seem unreasonable. The enhancement is due to multiple photon absorption by the quasiparticles i.e. both signal and probe, and the effect is most-pronounced near $h \nu_{\text {signal }}=2 \Delta$. This results in a higher average excess quasiparticle energy when the readout power is present alongside the signal compared to when only the signal is present, and also produces the small variation in $\eta$ between materials. The absorbed readout power $P_{\text {probe }}$ and readout frequency $h \nu_{\text {probe }}$ are not scaled by material parameters in this figure, unlike the signal. When calculated with $P_{\text {probe }}=0$, the signal quasiparticle generation efficiency is identical for all materials and is unity at $h \nu_{\text {signal }}=2 \Delta$.

Figure 6 shows the variation in $\eta$ as the bath temperature is changed. As $T_{b}$ increases, the scattering between the more numerous thermal quasiparticles and phonons determines the structure of the driven distribution, rather than scattering between the excess quasiparticles and phonons. This means the power flow from phonons to bath does not have the same peaked structure and instead more closely resembles the difference between two thermal distributions, as earlier shown in figure 2, This type of excess quasiparticle distribution means the average energy of generated quasiparticles increases without increasing the recombination and pair breaking rates. The figure also indicates temperatures for which $k_{B} T_{b}=2 \Delta\left(T_{b}\right)$ and $=\Delta\left(T_{b}\right)$, above which we expect thermal and nonequilibrium distributions to interact strongly. As $T_{b} / T_{c} \rightarrow 1$ we see that $\eta \rightarrow 1$ in agreement with the two-temperature model valid in that regime. We find that $T_{N}^{*}$ is wellaccounted for by (11), with the value of $\eta$ shown, for $T_{b} / T_{c} \leq 0.7$. The inset shows $T_{N}^{*}$ calculated using (11) for $\tau_{l} / \tau_{0}^{\phi}=1$ as a function of $P_{\text {signal with }} T_{b}=0.1 T_{c}$.

Figure 7 shows the dependence of $\eta$ on the phonon trapping factor $\tau_{l} / \tau_{p b}$ for $T_{b}=0.1 T_{c}$. As the phonon escape time $\tau_{l} / \tau_{0}^{\phi}$ increases, the quasiparticle generation efficiency $\eta \rightarrow 0.6$ for all materials examined. This con- 


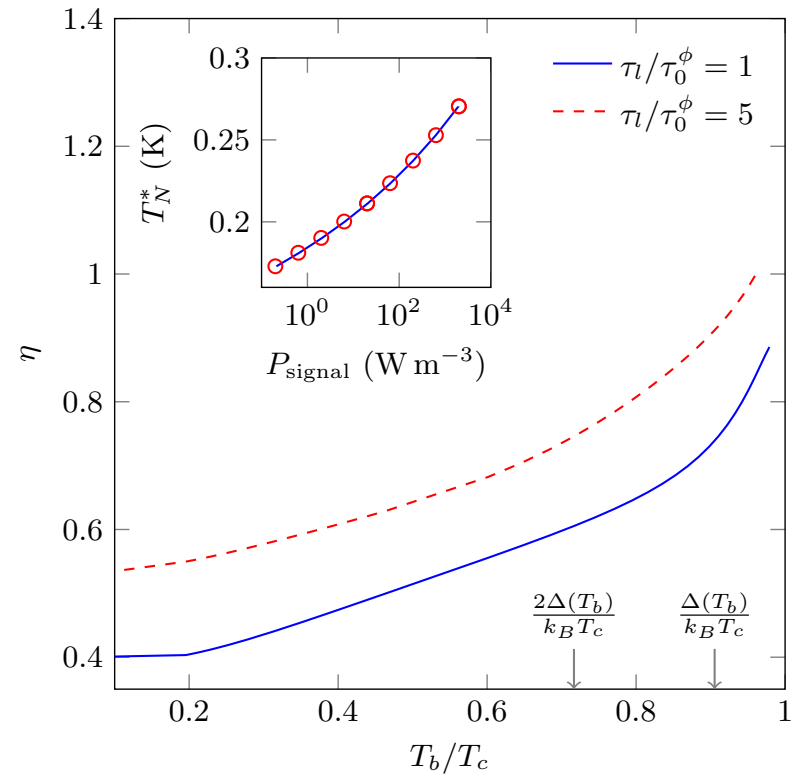

Figure 6. The quasiparticle generation efficiency $\eta$ as a function of reduced temperature $T_{b} / T_{c}$. The calculation is for for $\mathrm{Al}$, with $P_{\text {probe }}=0, P_{\text {signal }}=2 \mathrm{~W} \mathrm{~m}^{-3}, h \nu_{\text {signal }}=10 \Delta\left(T_{b}\right)$, and two values of the phonon escape time. The inset shows $T_{N}^{*}$ calculated using the full nonequilibrium model (red $\bigcirc$ ) and using (11) (blue line) as a function of $P_{\text {signal }}$ with $T_{b}=0.1 T_{c}$ and $\tau_{l} / \tau_{0}^{\phi}=1$.

clusion is in agreement with earlier Monte Carlo calculations $\frac{20 \mid 22}{2}$ for infinite superconductors or where $\Omega \geq 2 \Delta$ phonon loss was ignored.

\section{CONCLUSIONS}

We have described solutions of the coupled kinetic equations that calculate steady-state nonequilibrium quasiparticle and phonon distributions in a number of technologically important superconducting thin-films driven by sub-gap and pair-breaking photons. In particular we consider low energy signal photon interactions $h \nu_{\text {signal }} \leq 10 \Delta$, where localised heating and gap-suppression can be ignored. We have calculated numerical parameters for these superconductors that can be used in a simple analytical expression to describe the power-flow between quasiparticles and phonons. This expression allows straight-forward estimates of the effective quasiparticle temperatures for both sub-gap and pair-breaking drives to approximate the nonequilibrium behaviour without resorting to a full numeric solution of the coupled kinetic equations. The analytical expression is shown to give a good account of full solutions of the detailed equations for a wide range of powers and bath temperatures. This is relevant for predicting the behaviour of thin-film nonequilibrium superconducting detectors. We defined a low-energy quasiparticle generation efficiency for constant absorbed pair-breaking power and calculated detailed numerical solutions as a

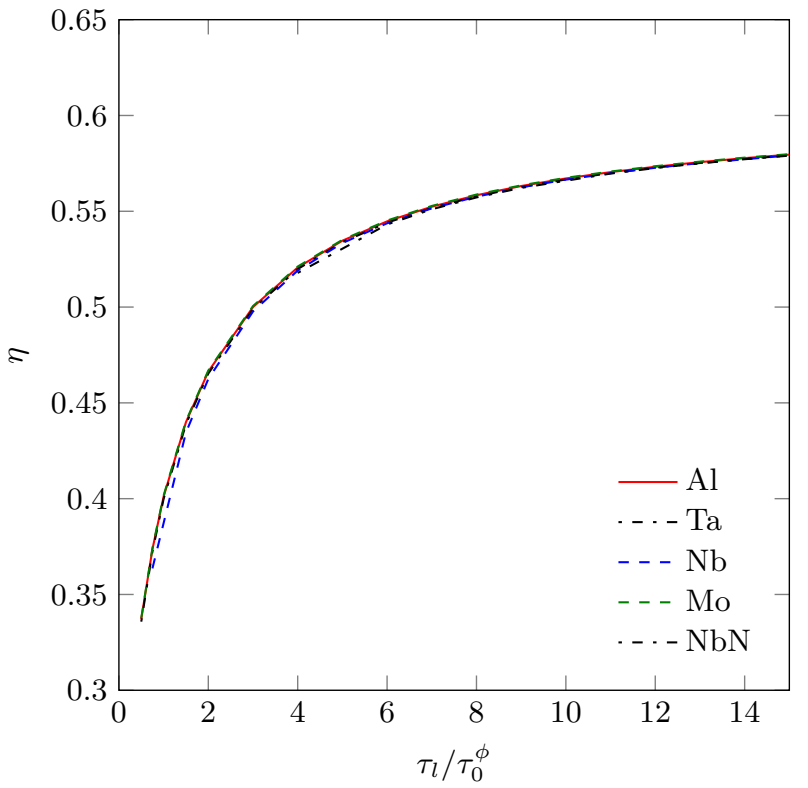

Figure 7. Quasiparticle generation efficiency $\eta$ for materials studied as a function of phonon escape time $\tau_{l} / \tau_{0}^{\phi}$. Calculation uses $T_{b}=0.1 T_{c}, P_{\text {probe }}=0, P_{\text {signal }}=2 \mathrm{~W} \mathrm{~m}^{-3}$, and $h \nu_{\text {signal }}=$ $10 \Delta$.

function of photon energies and bath temperature. We have shown that key parameters determining quasiparticle generation efficiency are the signal frequency and the phonon trapping factor. The enhancement of signal quasiparticle generation efficiency by absorbed readout power is demonstrated and explained as multiple-photon absorption (signal and probe) by the quasiparticles and Cooper pairs. We estimate this effect can result in an increase $\eta$ of up to $20 \%$ at signal photon energies of $h \nu_{\text {signal }}=2 \Delta$. The most sensitive detectors currently in development may also be able to distinguish between phonon trapping factors using results presented here. For the low-energy photon interactions studied we also show that the quasiparticle generation efficiency is material independent.
* tg307@mrao.cam.ac.uk

1 D. Mattis and J. Bardeen, Phys. Rev. 111, 412 (1958).

2 J. Bardeen, G. Rickayzen, and L. Tewordt, Phys. Rev. 113, $982(1959)$.
3 J. Zmuidzinas, Annu. Rev. Condens. Matter Phys. 3, 169 (2012)

4 J. Baselmans, J. Low Temp. Phys. 167, 292 (2012) 
${ }^{5}$ G. Vardulakis, S. Withington, D. J. Goldie, and D. M. Glowacka, Meas. Sci. Technol. 19, 015509 (2008).

6 S. Friedrich, J. Low Temp. Phys. 151, 277 (2008).

7 L. DiCarlo, M. D. Reed, L. Sun, B. R. Johnson, J. M. Chow, J. M. Gambetta, L. Frunzio, S. M. Girvin, M. H. Devoret, and R. J. Schoelkopf, Nature 467, 574 (2010)

8 M. Hofheinz, E. M. Weig, M. Ansmann, R. C. Bialczak, E. Lucero, M. Neeley, A. D. O'Connell, H. Wang, J. M. Martinis, and A. N. Cleland, Nature 454, 310 (2008)

9 R. J. Schoelkopf and S. M. Girvin, Nature 451, 664 (2008).

${ }^{10}$ K. D. Irwin and K. W. Lehnert, Appl. Phys. Lett. 85, 2107 (2004)

1 J. Bueno, M. D. Shaw, P. K. Day, and P. M. Echternach, Appl. Phys. Lett. 96, 103503 (2010).

12 B. I. Ivlev, S. G. Lisitsyn, and G. M. Eliashberg, J. Low Temp. Phys. 10, 449 (1973)

13 A. Zehnder, Phys. Rev. B 52, 12858 (1995)

14 A. D. Semenov, G. N. Gol'tsman, and R. Sobolewski, Supercond. Sci. Technol. 15, R1 (2002).

15 A. D. Semenov, R. S. Nebosis, Y. P. Gousev, M. A. Heusinger, and K. F. Renk, Phys. Rev. B 52, 581 (1995).

16 W. Parker, Phys. Rev. B 12, 3667 (1975).

17 C. Owen and D. Scalapino, Phys. Rev. Lett. 28, 1559 (1972)

J.-J. Chang and D. J. Scalapino, Phys. Rev. B 15, 2651 (1977)

9 J.-J. Chang and D. J. Scalapino, J. Low Temp. Phys. 31, 1 (1978)

${ }^{20}$ M. Kurakado, Nucl. Instrum. Methods 196, 275 (1982)

21 N. Rando, A. Peacock, A. van Dordrecht, C. Foden, R. Engelhardt, B. Taylor, P. Gare, J. Lumley, and C. Pereira, Nucl. Instruments Methods 313, 173 (1992)

22 R. A. Hijmering, P. Verhoeve, D. D. E. Martin, A. G. Kozorezov, J. K. Wigmore, R. Venn, P. J. Groot, and I. Jerjen, J. Appl. Phys. 105, 123906 (2009).

23 A. G. Kozorezov, A. F. Volkov, J. K. Wigmore, A. Peacock, A. Poelaert, and R. den Hartog, Phys. Rev. B 61, 11807 (2000)

${ }^{24}$ D. J. Goldie and S. Withington, Supercond. Sci. Technol. 26, $015004(2013)$

25 T. Guruswamy, D. J. Goldie, and S. Withington, Supercond. Sci. Technol. 27, 055012 (2014), arXiv:1401.1937

20 P. J. de Visser, D. J. Goldie, P. Diener, S. Withington, J. J. A. Baselmans, and T. M. Klapwijk, Phys. Rev. Lett. 112, 047004 (2014), arXiv:1306.4992

27 P. J. de Visser, J. J. A. Baselmans, J. Bueno, N. Llombart, and T. M. Klapwijk, Nat. Commun. 5, 3130 (2014).

28 A. Neto, N. Llombart, J. J. A. Baselmans, A. Baryshev, and S. J. C. Yates, IEEE Trans. Terahertz Sci. Technol. 4,
$26(2014)$

29 C. C. Chin, D. E. Oates, G. Dresselhaus, and M. Dresselhaus, Phys. Rev. B 45, 4788 (1992).

30 S. Kaplan, C. Chi, D. Langenberg, J.-J. Chang, S. Jafarey, and D. Scalapino, Phys. Rev. B 14, 4854 (1976)

31 J. Caro, R. Coehoorn, and D. de Groot, Solid State Commun. 39, 267 (1981)

${ }^{32}$ K. Kihlstrom, R. Simon, and S. Wolf, Phys. Rev. B 32, $1843(1985)$

${ }^{3}$ A. Semenov, B. Günther, U. Böttger, H.-W. Hübers, H. Bartolf, A. Engel, A. Schilling, K. Ilin, M. Siegel, R. Schneider, D. Gerthsen, and N. Gippius, Phys. Rev. B 80, 054510 (2009)

34 E. M. Gerhsenzon, M. E. Gershenzon, G. N. Golt'tsman, A. M. Lyul'kin, and A. V. Sergeev, Sov. Phys. JETP 70, 505 (1990).

35 G. Gladstone, M. Jensen, and J. Schrieffer, in Superconductivity, Vol. 2, edited by R. D. Parks (M. Dekker, New York, 1969) Chap. 13, p. 713.

36 Y. P. Gousev, G. N. Golt'tsman, A. D. Semenov, E. M. Gerhsenzon, R. S. Nebosis, M. A. Heusinger, and K. F. Renk, J. Appl. Phys. 75, 3695 (1994).

37 A. G. Kozorezov, J. Low Temp. Phys. 167, 473 (2011).

38 E. Gershenzon, G. Gol'tsman, V. Potapov, and A. Sergeev, Solid State Commun. 75, 639 (1990)

39 This differs by a factor of $\pi$ from the expression given in Altshuler and Aronov 47 .

${ }^{40}$ A. V. Sergeev and M. Yu. Reizer, Int. J. Mod. Phys. B 10, 635 (1996)

${ }^{41}$ P. J. de Visser, J. J. A. Baselmans, S. J. C. Yates, P. Diener, A. Endo, and T. M. Klapwijk, Appl. Phys. Lett. 100, 162601 (2012)

${ }^{42}$ L. E. Toth, Transition metal carbides and nitrides, Refractory materials, Vol. 7 (Academic Press, New York, 1971) p. 279 .

43 K. Schwarz, J. Phys. C Solid State Phys. 8, 809 (1975)

44 G. M. Eliashberg, Sov. Phys. - JETP 34, 668 (1972).

45 L. Parlato, R. Latempa, G. Peluso, G. Pepe, R. Cristiano, and R. Sobolewski, Supercond. Sci. Technol. 18, $1244(2005)$

46 A. Rothwarf and B. N. Taylor, Phys. Rev. Lett. 19, 27 (1967)

B. Altshuler and A. Aronov, in Electron-Electron Interact. Disord. Syst. Modern Problems in Condensed Matter Sciences, Vol. 10, edited by A. Efros and M. Pollak (North Holland, Amsterdam, New York, 1985) Chap. 1, pp. 1-153. 